Infect Disord Drug Targets. 2020;20(1):49-55.

doi: $10.2174 / 1871526519666190206205521$.

\title{
Plasmid-Mediated Quinolone Resistance in Pseudomonas aeruginosa Isolated from Burn Patients in Tehran, Iran
}

Azam Molapour 1, Amir Peymani 2, Parvaneh Saffarain 1, Narges Habibollah-Pourzereshki 2, Pooya Rashvand 3

${ }^{1}$ Department of Microbiology, Science and Research Branch, Islamic Azad University, Tehran, Iran.

${ }^{2}$ Medical Microbiology Research Center, Qazvin University of Medical Sciences, Qazvin, Iran.

${ }^{3}$ Student Research Committee, Qazvin University of Medical Sciences, Qazvin, Iran.

\begin{abstract}
Introduction: Plasmid-induced quinolone resistance has raised a great concern in the treatment of serious infections worldwide. The aims of this study were to determine the antibiotic susceptibility, the frequency of qepA, aac(6')-Ib and qnr genes by PCR and sequencing, and typing of the resistant isolates using repetitive extragenic palindromic sequence-based PCR (REPPCR) in Pseudomonas aeruginosa isolated from burn wound infections.

Methods: In the current cross-sectional study, 149 P. aeruginosa were isolated from the burn wound samples of patients admitted to Motahari hospital in Tehran, Iran, from February to December 2016. The bacterial isolates were identified using standard laboratory methods and their antibiotic susceptibility to quinolones was evaluated using the standard Kirby-Bauer method, according to the Clinical and Laboratory Standards Institute (CLSI) guidelines. The presence of aac(6')-Ib, qepA, qnrA, qnrB4, qnrB and qnrS genes was assessed using PCR and sequencing methods and clonal relationship of the resistant isolates was evaluated using REP-PCR method.

Results: All (100\%) isolates showed complete resistance to used quinolone compounds in this study. The qnr and qepA genes were not found, but all (100\%) isolates were positive for the presence of aac $\left(6^{\prime}\right)-\mathrm{Ib}$ gene and the sequencing revealed that all $(100 \%)$ belong to the aac $\left(6^{\prime}\right)-\mathrm{Ib}$ cr variant. REP-PCR showed that the studied isolates belonged to three distinct clones of A (77.9\%), B (18.1\%), and C (4\%).

Conclusion: The findings of the present study indicated the presence of aac $\left(6^{\prime}\right)$-Ib-cr variant and lack of the contribution of qnr and qepA in the emergence of resistance to quinolones in $\mathrm{P}$. aeruginosa isolated from burn patients. Considering the importance of clonal spread of these resistant isolates and their significant role in the development of clinical infections, especially in patients with burns, more attention should be paid to the prevention of the dissemination of these resistant isolates.
\end{abstract}

Keywords: PCR; Pseudomonas aeruginosa; aac(6')-Ib; gram-negative pathogens; plasmidmediated quinolone resistance; qepA; qnr 